\title{
POLYNOMIALS AND THE LIMIT POINT CONDITION
}

\author{
BY
}

\author{
ROBERT M. KAUFFMAN
}

\begin{abstract}
An $n$th order, possibly nonselfadjoint, ordinary differential expression $L$ is said to be in the limit point condition if the maximal operator $L_{M}$ in $L_{2}[0, \infty)$ is an $n$-dimensional extension of the minimal operator $L_{0}$. If range $L_{0}$ is closed, this definition is equivalent to the assertion that nullity $L_{M}+$ nullity $\left(L^{+}\right)_{M}=n$, where $L^{+}$is the formal adjoint of $L$. It also implies that any operator $T$ such that $L_{0} \subseteq T \subseteq L_{M}$ is the restriction of $L_{M}$ to a set of functions described by a boundary condition at zero. In this paper, we discuss the question of when differential expressions involving complex polynomials in selfadjoint expressions are in the limit point condition.
\end{abstract}

1. Introduction. In this paper we study the property that a polynomial in a selfadjoint ordinary differential expression is in the limit point condition. We extend the definition of the limit point condition in a natural way to nonselfadjoint expressions, and can thus study complex as well as real polynomials. Many of our theorems also hold, with the same proof, for vector matrix differential expressions.

We shall say that an ordinary differential expression $\tau$ is in the limit point condition if the maximal operator $\tau_{M}$ in $L_{2}[0, \infty)$ is an $n$-dimensional extension of the minimal operator $\tau_{0}$, where $n$ is the order of $\tau$. This means that there are no "boundary conditions at infinity," so that any operator in between $\tau_{0}$ and $\tau_{M}$ is specified by a boundary condition at zero. It turns out that, if range $\tau_{0}$ is closed, $\tau$ is in the limit point condition if and only if the number of linearly independent solutions in $L_{2}[0, \infty)$ to the equation $\tau f=0$, plus the corresponding number for the adjoint equation $\tau^{+} f=0$, equals the order of $\tau$.

It is proved in this paper that if any $n$th degree complex polynomial in any formally selfadjoint ordinary differential expression $\tau$ is in the limit point condition, then all polynomials in $\tau$ of degree less than or equal to $n$ are in the limit point condition. In particular, $\tau$ itself which can have complex coefficients and be of arbitrary order must be in the limit point condition. This proves, in a very

Presented to the Society, January 17,1974 ; received by the editors November 8 , 1973 and, in revised form, February 19, 1974.

Key words and phrases. Limit point condition, minimal operator, maximal operator, Fredholm operator, perturbation theory, product of operators, complex polynomials in a formally selfadjoint expression. 
general situation, a conjecture of Everitt and Giertz [3] about second order $\tau$, that if $\tau$ and $\tau^{n}$ are in the limit point condition, $\tau^{j}$ is also in the limit point condition for $1<j<n$. In addition, the equivalence of "partial separation" and the limit point condition is proved for any complex polynomial in any selfadjoint $\tau$, provided that $\tau$ is in the limit point condition. This generalizes results of Everitt and Giertz [3] and of Zettl [10].

Also, simple growth conditions on the derivative of $V$ are given to assure that a complex polynomial in $\tau$ is in the limit point condition, where $\tau=$ $-d^{2} / d x^{2}+V$ or $i d / d x+V$, and $V$ is real valued and $C^{\infty}$. In the second order case, we must assume also that $V$ is bounded below. The growth condition is that, for some $k>0, e^{-k x} V^{(i)}(x)$ is a bounded function on $[0, \infty)$ for all derivatives $V^{(i)}$ with $i \leqslant n-2$ in the first order case, and $i \leqslant 2 n-4$ in the second order case, where $n$ is the degree of the polynomial. Finally, it is shown that, when $\tau=i d / d x+V$ and $V$ is real valued and satisfies the above growth condition, $\tau^{n}+\Sigma_{0}^{n-1} f_{m} \tau^{m}$ is in the limit point condition, provided that the first $m$ derivatives of each complex valued function $f_{m}$ are bounded.

All the results except the results concerning first and second order $\tau$ extend, with the same proof, to vector matrix differential expressions. The methods of proof are new, and use the perturbation theory of unbounded Fredholm operators. It should be mentioned, however, that while the methods of proof are new, the use of perturbation theorems to analyze the limit point condition is not.

Chaudhuri and Everitt [1] proved the original theorems of the type studied in this paper, for squares of certain second order expressions. They also give an example of a second order $\tau$, in the limit point condition, such that $\tau^{2}$ is not in the limit point condition. Everitt and Giertz [3] studied the $n$th powers of certain second order exprssions, and introduced the "partial separation" conditions. They proved that $\left(-d^{2} / d x^{2}+V\right)^{n}$ is in the limit point condition provided that $V$ is real valued and either (a) $V(x) \geqslant k>0$ and the second derivative of $V$ satisfies a nonoscillation condition, or (b) $V$ differs from a real constant by an $L_{1}$ function.

Zettl [10] generalized the partial separation results of Everitt and Giertz to powers in arbitrary selfadjoint differential expressions with real coefficients. He also generalized condition (b) cited above of Everitt and Giertz to arbitrary order selfadjoint $\tau$ with real coefficients. Finally, a corollary of Zettl's results is that, for a $\tau$ with real coefficients which is in the limit point condition, then a real polynomial $\tau^{n}+\Sigma_{0}^{n-1} c_{i} \tau^{i}$ is in the limit point condition if and only if $\tau^{n}$ is in the limit point condition. Zettl's results, however, do not say anything above lower degree polynomials.

This paper does not use the results or methods of Everitt, Giertz and Zettl, but is of course motivated by their results. The author would like to express his 
appreciation to A. Zettl for much helpful correspondence regarding this paper.

\section{Polynomials in $n$th order selfadjoint expressions.}

DEFinition. We say that $L$ is a regular ordinary differential expression if $L$ is of form $\Sigma_{0}^{n} f_{i} D^{i}$ where $D=d / d x$, and each $f_{i}$ is a $\dot{C}^{\infty}$ complex valued function, defined in $[0, \infty)$, with $f_{n}$ nonvanishing. (We assume $C^{\infty}$ coefficients in order to take.powers and adjoints of differential expressions freely.)

REMARK. When we refer to a differential expression, or to an operator, in this paper, we always mean a linear differential expression or operator.

Notation. Let $L$ be a regular ordinary differential expression. Then $L_{0}$ denotes the minimal operator associated with $L$ in $L_{2}[0, \infty)$, and $L_{M}$ denotes the maximal operator. $L^{+}$denotes the formal adjoint $\Sigma_{0}^{n}(-1)^{i} D^{i-\theta} f_{i}$ of $L$. (See Dunford and Schwartz [2], Goldberg [5], or Naimark [8] for an account of these concepts.)

REMARK. If $L$ is a regular ordinary differential expression of order $n$ with $C^{\infty}$ coefficients on the interval $[0, \infty)$, and $L=L^{+}$, then $L$ can be written in the form

$$
L f=\sum_{0}^{[n / 2]}\left(p_{j} f^{(j)}\right)^{(j)}+i \sum_{0}^{[(n-1) / 2]}\left[\left(q_{j} f^{(j)}\right)^{(j+1)}+\left(q_{j} f^{(j+1)}\right)^{(j)}\right]
$$

where $p_{j}$ and $q_{j}$ are real $C^{\infty}$ functions and the leading coefficient $p_{[n / 2]}$ or $q_{[(n-1) / 2]}$ is nonzero on $[0, \infty)$. This is shown in Dunford and Schwartz [2].

DEFinition. Let $T$ be a closed, densely defined operator from a Hilbert space $h$ into itself with a finite-dimensional null space and a closed range which has finite deficiency in $h$. Then $T$ is called a Fredholm operator. The index of $T$ is defined to be the nullity of $T$ minus the deficiency of range $T$ in $h$.

Definition. Suppose that $T_{2}$ and $T_{1}$ are linear operators. Suppose that domain $T_{2} \subseteq$ domain $T_{1}$, and $T_{2}$ and $T_{1}$ agree on domain $T_{2}$. Then $T_{1}$ is said to be an extension of $T_{2}$. If $T_{1}$ is an extension of $T_{2}$, and the dimension of the quotient vector space domain $T_{1} /$ domain $T_{2}$ is exactly $n$, then $T_{1}$ is said to be an $n$-dimensional extension of $T_{2}$.

REMARK. If $T_{2}$ is a Fredholm operator and $T_{1}$ is an $n$-dimensional extension of $T_{2}$, then $T_{1}$ is a Fredholm operator and index $T_{1}=\operatorname{index} T_{2}+n$.

The following theorems are well known. (See Naimark [8] for the formally selfadjoint case, or Dunford and Schwartz [2], or Goldberg [5] for the general case. The vector matrix case can be proved by using similar methods to those in these books.)

THEOREM 1. The following are equivalent: (a) $L_{0}$ has closed range; (b) $\left(L^{+}\right)_{0}$ has closed range; (c) $L_{M}$ has closed range. 
THEOREM 2. $\left(L_{0}\right)^{*}=\left(L^{+}\right)_{M}$, where $\left(L_{0}\right)^{*}$ denotes the adjoint of $L_{0}$ in $L_{2}[0, \infty)$.

THEOREM 3. If $f$ is in domain $L_{0}$, and $L$ is nth order, then $D^{i} f(0)=0$ for $0 \leqslant i \leqslant n-1$. Thus $L_{0}$ has no null space except the zero vector.

THEOREM 4. If $L_{0}$ has closed range then $L_{M}$ is surjective.

The following theorems, which we shall use repeatedly, are proved in Goldberg [5]. Theorem 5 is obvious, though it is worthwhile to state it. Theorem 6 is a special case of a very powerful result basically due to Kato [6]

THEOREM 5. Let $T$ be a Fredholm operator. Then $T^{*}$ is a Fredholm operator, and index $T^{*}=-$ index $T$.

Definition. Let $S$ be a closed subspace of a Hilbert space $h$. Let $d(x, S)$ denote the distance from $x$ to $S$. Let $T$ be an operator in $h$. Then $\gamma(T)$, the minimum modulus of $T$, is defined to be $\inf \|T x\| / d(x, N(T))$, where $N(T)$ is the null space of $T$, and the inf is taken over all $x$ in domain $T$, and where $0 / 0$ is taken to be $\infty$. If $T$ is a Fredholm operator, $\gamma(T)>0$.

A discussion of the next theorem is found on p. 122 of Goldberg [5].

THEOREM 6. Let $T$ be a Fredholm operator in a Hilbert space $h$. Suppose that $a$ and $b$ are nonnegative real numbers such that $a+b \gamma(T)<\gamma(T)$. Suppose $A$ is an operator in $h$, with domain $A \supseteq$ domain $T$, and $\|A x\| \leqslant a\|x\|+$ $b\|T x\|$ for every $x$ in domain $T$. Then $T+A$ is a Fredholm operator, index $T+A$ is the same as index $T$, the nullity of $T+A$ is less than or equal to the nullity of $T$, and the deficiency of range $T+A$ in $h$ is less than or equal to the deficiency of range $T$.

Remark. Recall that $T+A$ is defined to be $(T+A) x$ for every $x$ in domain $(T+A)$, and domain $(T+A)$ is defined to be domain $T \cap$ domain $A$.

Definition. Let $T$ be a closed operator in a Hilbert space $h$, and let $A$ be an operator in $h$ with domain $A \supseteq$ domain $T$. Then $A$ is said to be $T$ bounded if, for some $M>0,\|A x\| \leqslant M(\|x\|+\|T x\|)$ for all $x$ in domain $T$.

REMARK. If $A$ and $T$ are closed, and domain $A \supseteq$ domain $T$, then it follows from the closed graph theorem that $A$ is $T$ bounded. Thus a small enough multiple of $A$ will fulfill the hypotheses of Theorem 6.

Definition. Let $L$ be an $n$th order regular ordinary differential expression. Then $L$ is said to be in the limit point condition if $L_{M}$ is an $n$-dimensional extension of $L_{0}$.

REMARK. If $L$ is in the limit point condition, then domain $L_{M}$ is the vector subspace (which is not closed) generated by domain $L_{0}$ and $n$ functions $f_{j}$, vanishing outside a closed interval $[0, N]$, such that $D^{i} f_{j}(0)=\delta_{i j}$ for all 
$0 \leqslant i \leqslant n-1$ and $0 \leqslant j \leqslant n-1$, where $\delta$ denotes the Kronecker delta. It is clear that all these functions are in domain $L_{M}$, and no nontrivial linear combination of them can be in domain $L_{0}$, since the first $n-1$ derivatives of elements of domain $L_{0}$ all vanish at zero. The conclusion follows.

THEOREM 7. Suppose that $L$ is an nth order regular ordinary differential expression. Suppose that $L_{0}$ has closed range. Then $L$ is in limit point condition if and only if nullity $L_{M}+\operatorname{nullity}\left(L^{+}\right)_{M}=n$.

Proof. Nullity $L_{M}=$ index $L_{M}=$ index $L_{0}+k$, where $L_{M}$ is a $k$-dimensional extension of $L_{0}$. But index $L_{0}=-$ deficiency of range $L_{0}=$ - nullity $\left(L^{+}\right)_{M}$. Thus nullity $L_{M}+\operatorname{nullity}\left(L^{+}\right)_{M}=k$.

CoROLlary 1. Suppose that $L$ is a regular differential expression such that $L_{0}$ has closed range. Then $L_{M}$ is a $k$-dimensional extension of $L_{0}$, where $k=$ nullity $L_{M}+\operatorname{nullity}\left(L^{+}\right)_{M}$.

Proof. Corollary 1 follows from the proof of Theorem 7.

Notation. Let $C_{0}^{\infty}$ denote the infinitely differentiable complex valued functions defined on $[0, \infty)$ which vanish outside a compact sabset of $(0, \infty)$. Thus in particular each element of $C_{0}^{\infty}$ vanishes in a neighborhood of zero.

COROLlary 2. Our definition of the limit point condition is equivalent to the usual one, when the differential expression is formally selfadjoint.

Proof. Let $z=(-1)^{1 / 2}$. A regular formally selfadjoint expression $L$ is usually said to be in the limit point condition if nullity $(L+z)_{M}+\operatorname{nullity}(L-z)_{M}$ $=n$, where $n$ is the order of $L$. This definition is equivalent to our definition, as we show.

In fact, if $L=L^{+}$, and $f$ is in $C_{0}^{\infty}$, then $(L f, f)$ is real, where $($, denotes the inner product in $L_{2}[0, \infty)$. Thus $|((L+z) f, f)| \geqslant(f, f)$ for any $f$ in $C_{0}^{\infty}$. Therefore, by the Schwarz inequality, $\|(L+z) f\| \geqslant\|f\|$ for all $f$ in $C_{0}^{\infty}$. Since $(L+z)_{0}$ is the closure of its restriction to $C_{0}^{\infty}$, it follows that $(L+z)_{0}$ has a bounded inverse from its range into $L_{2}[0, \infty)$. Since $(L+z)_{0}$ is a closed operator, its range must therefore also be closed. By Corollary 1 , nullity $(L+z)_{M}+\operatorname{nullity}(L-z)_{M}=k$, where $(L+z)_{M}$ is a $k$-dimensional extension of $(L+z)_{0}$. Thus $L+z$ is in our limit point condition if and only if $k=n$, or in other words, if and only if $L$ is in the usual limit point condition.

We need to show that domain $(L+z)_{M}=$ domain $L_{M}$, and domain $(L+z)_{0}$ $=$ domain $L_{0}$. This will show that the addition of $z$ to $L$ does not change the limit point condition.

We show that for any regular differential expression $L$, whether or not $L=L^{+}$, and for any complex constant $c$, domain $(L+c)_{M}=$ domain $L_{M}$, 
and domain $(L+c)_{0}=$ domain $L_{0}$. The first equality is obvious. The second is a consequence of the fact that both $L_{0}$ and $(L+z)_{0}$ are the closure of their restrictions to $C_{0}^{\infty}$. In fact, if $f$ is in domain $L_{0}$, there is a sequence $f_{n}$ in $C_{0}^{\infty}$ with $f_{n}$ converging to $f$ and $L f_{n}$ converging to $L f$. Thus $(L+c) f_{n}$ converges to $(L+c) f$, so $f$ is in domain $(L+c)_{0}$. Reversing the argument, we see that the domains are equal. Lemma 2 is proved.

COROLlaRy 3. Let $L$ be an nth order, regular, ordinary differential expression, and suppose that range $L_{0}$ is closed. Then nullity $L_{M}+\operatorname{nullity}\left(L^{+}\right)_{M} \geqslant n$.

Proof. $L_{M}$ is clearly at least an $n$-dimensional extension of $L_{0}$, as may be seen from considering the functions of the remark preceding Theorem 7. The corollary then follows.

REMARK. The range of $L_{0}$ is closed in $L_{2}[0, \infty)$ for a large number of differential expressions $L$. It was previously shown that range $(L+z)_{0}$ is closed if $L=L^{+}$, but the range of the minimal operator is closed in many other situations. For example, suppose that $(L f, f)$ is bounded away from zero for all $f$ in $C_{0}^{\infty}$ with $\|f\|=1$. Then, as in Corollary 2 above, it follows from the Schwarz inequality that $\left(L_{0}\right)^{-1}$ is a bounded linear transformation from its range into $L_{2}[0, \infty)$, so that, since $L_{0}$ is a closed operator, range $L_{0}$ is closed. Thus, for example, if $L=\left(L_{1}\right)^{+} L_{1}$, and the real part of $f$ is positive and bounded away from zero, $(L+f)_{0}$ has closed range. Also, if $L$ is formally selfadjoint, and $L=L^{+}$, then $(L+f)_{0}$ has closed range if the imaginary part of $f$ is bounded away from zero. Once considerations like this establish that $L_{0}$ has closed range, Theorem 6 can be used to show that, for a wide class of differential expressions $B,(L+B)_{0}$ has closed range, even though $((L+B) f, f)$ may take on the value zero for $f$ in $C_{0}^{\infty}$.

The preceding examples are rather elementary, but they illustrate one of the most common techniques of showing that $L_{0}$ has closed range. Some more sophisticated examples will appear in the sequel. Another simple way of showing that $L_{0}$ has closed range follows.

LEMma 1. Suppose that $L=L_{1} L_{2}$, where $L_{1}$ and $L_{2}$ are regular ordinary differential expressions and $\left(L_{1}\right)_{0}$ and $\left(L_{2}\right)_{0}$ have closed range. Then $L_{0}$ has closed range.

Proof. Since each $\left(L_{i}\right)_{0}$ has closed range, it follows from the closed graph theorem that each $\left(L_{i}\right)_{0}$ has a bounded inverse from its range into $L_{2}[0, \infty)$. Now $L_{0}$ is the closure of its restriction to $C_{0}^{\infty}$. Further, $L_{1} L_{2}$, when restricted to $C_{0}^{\infty}$, has a bounded inverse from its range into $L_{2}[0, \infty)$, since the product of bounded operators is bounded. Passing to the closure, we see that $L_{0}$ has a 
bounded inverse from its range into $C_{0}^{\infty}$. Since $L_{0}$ is closed, this means that range $L_{0}$ is closed.

THEOREM 8. Suppose that $\tau$ is a regular formally selfadjoint ordinary differential expression. Suppose that $\tau^{j}+\Sigma_{0}^{j-1} c_{i} \tau^{i}$ is in the limit point condition, where each $c_{i}$ is a complex number. Then for any $k \leqslant j$, and any $(k+1)$-tuple of complex numbers $\left\langle d_{0}, d_{1}, \cdots, d_{k}\right\rangle, \Sigma_{0}^{k} d_{i} \tau^{i}$ is in the limit point condition.

REMARK. We do not assume that $\tau$ is in the limit point condition. This follows as a consequence of the theorem.

Before proceeding with the proof of Theorem 8, a number of lemmas are needed. These lemmas will be used also in the proofs of later theorems.

LEMMA 2. Let $\left\{L_{i}\right\}$ be a set of regular ordinary differential expressions. Suppose that each $\left(L_{i}\right)_{0}$ has closed range. Then, if $L=L_{1} L_{2} \cdots L_{n}$ is in the limit point condition, each $L_{i}$ is in the limit point condition.

Proof of Lemma 2. Let $n_{i}$ be the order of $L_{i}$. Since each $\left(L_{i}\right)_{M}$ is surjective, it follows that nullity $L_{M} \geqslant \Sigma_{i}^{j}$ nullity $\left(L_{i}\right)_{M}$. To see this, one need only verify it for the case of two operators and proceed by induction, since the composition of surjective operators is surjective. But if $T_{1}$ and $T_{2}$ are any surjective linear transformations, we can see from dividing out the null space of $T_{2}$ that there are $k$ vectors $f_{i}$ in domain $T_{2}$, which are linearly independent modulo the null space of $T_{2}$, such that $T_{2}\left(f_{i}\right)=g_{i}$, where the $g_{i}$ form a basis for the $k$-dimensional null space of $T_{1}$. Thus nullity $T_{1} T_{2}=$ nullity $T_{1}+$ nullity $T_{2}$. Similarly, nullity $\left(L^{+}\right)_{M} \geqslant \Sigma_{i}^{j}$ nullity $\left(L_{i}^{+}\right)_{M}$. Since $L_{0}$ has closed range, by Lemma 1 , it follows that $L$ is in the limit point condition if and only if nullity $L_{M}+\operatorname{nullity}\left(L^{+}\right)_{M}=\Sigma_{1}^{j} n_{i}$. Since by Corollary 3 to Theorem 7, nullity $\left(L_{i}\right)_{M}+\operatorname{nullity}\left(L_{i}^{+}\right)_{M} \geqslant n_{i}$, the conclusion follows.

Lemma 2 can be used in the proof of Theorem 8 to pass from $n$th degree to lower degree polynomials. However, the polynomial we are studying may not be the product of factors $\tau+\lambda_{i}$ such that $\left(\tau+\lambda_{i}\right)_{0}$ has closed range. What is needed is a way to go from a simple polynomial such as $(\tau+z)^{j}$, where $z=$ $(-1)^{1 / 2}$, to the polynomial we are dealing with. We use Theorem 6 to do this, but we must lay some groundwork. Lemmas 4 and 5 do this, in a somewhat more general setting than needed for Theorem 8 . The more general setting is needed for later theorems.

We first need a functional analytic lemma. This is Theorem 2 of Kauffman [7]. 
LEMma 3. Let $H$ be a selfadjoint operator in a Hilbert space $h$. Suppose that $f_{n}$ is a sequence in domain $H^{2 j}$ with $\left\|f_{n}\right\|=1$ and $\left|\left(H^{i} f_{n}, f_{n}\right)\right|$ approaching infinity for some $i<2 j$. Then $\left(H^{i} f_{n}, f_{n}\right) /\left(H^{2 j} f_{n}, f_{n}\right)$ converges to zero as $n$ approaches infinity.

COROLlary. Let $H$ be a selfadjoint operator in a Hilbert space h. Suppose that $f_{n}$ is a sequence, with $\left\|f_{n}\right\|=1$, in domain $H^{2 j}$ such that, for some $i<2 j$, $\left|\left(H^{i} f_{n}, f_{n}\right)\right|$ approaches infinity. Then, for all $k<2 j,\left(H^{k} f_{n}, f_{n}\right) /\left(H^{2 j} f_{n}, f_{n}\right)$ approaches zero as $n$ approaches infinity.

PROOF. $\left(H^{k} f_{n}, f_{n}\right)$ is either a bounded sequence or it has a subsequence with absolute value converging to infinity. If it is bounded, the conclusion clearly follows from Lemma 3. However, for any subsequence $\left(H^{k} f_{n_{j}}, f_{n_{j}}\right)$ which converges in absolute value to infinity, Lemma 3 applies to force $\left(H^{k} f_{n_{j}}, f_{n_{j}}\right) /\left(H^{2 j} f_{n}, f_{n}\right)$ to converge to zero. Thus in no case can a subsequence of $\left(H^{k} f_{n}, f_{n}\right) /\left(H^{2 j} f_{n}, f_{n}\right)$ be bounded away from zero. The corollary is proved.

LEMMA 4. Suppose $\tau$ is a regular formally selfadjoint ordinary differential expression. Let $L=\tau^{j}+\Sigma_{0}^{j-1} g_{i} \tau^{i}$, where each $g_{i}$ is a bounded $C^{\infty}$, complex valued function. Then the restriction of $\tau^{i}$ to domain $L_{0}$ is $L_{0}$ bounded for any $i \leqslant j$.

REMARK. Of course, we cannot even assume without proof that $\tau^{i} f$ is in $L_{2}[0, \infty)$ if $f$ is in domain $L_{0}$. This is in fact equivalent to what we wish to prove.

Proof of Lemma 4. We first show that there is an $M>0$ such that $M(\|f\|+\|L f\|) \geqslant\left\|\tau^{i} f\right\|$ for all $f$ in $C_{0}^{\infty}$ and each $i$ with $j \geqslant i \geqslant 1$. It suffices to consider $i<j$.

Suppose the proposition is not true. Then there is a sequence $f_{n}$ in $C_{0}^{\infty}$ with $\left\|f_{n}\right\|=1$ and $\left\|\tau^{i} f_{n}\right\|>n\left(\left\|f_{n}\right\|+\left\|L f_{n}\right\|\right) . \tau_{0}$, being symmetric, has a selfadjoint extension $H$, perhaps to a larger Hilbert space. We then have

$$
\left\|H^{i} f_{n}\right\|^{2}>n^{2}\left(\left\|f_{n}\right\|^{2}+2\left\|f_{n}\right\|\left\|L f_{n}\right\|+\left\|L f_{n}\right\|^{2}\right) \text {. }
$$

Since $\left\|f_{n}\right\|=1$, it follows that $\left(H^{2} i_{f_{n}}, f_{n}\right)>n^{2}$. By the corollary to Lemma 3, it follows that, for any $k<2 j,\left(H^{k} f_{n}, f_{n}\right) /\left(H^{2 j} f_{n}, f_{n}\right)$ converges to zero as $n$ approaches infinity. Thus $\left\|L f_{n}\right\|^{2} /\left(H^{2 j} f_{n}, f_{n}\right)$ approaches 1 . Dividing both sides of (1) by $\left(H^{2 j} f_{n}, f_{n}\right)$, we obtain a contradiction.

Therefore we know that there is an $M>0$ such that, for all $f$ in $C_{0}^{\infty}$, $M(\|f\|+\|L f\|) \geqslant\left\|\tau^{i} f\right\|$. We must extend this inequality to all $f$ in domain $L_{0}$.

Recall that $L_{0}$ is the closure of its restriction to $C_{0}^{\infty}$. Therefore, if $\langle f, L f\rangle$ is in the graph of $L_{0}$, there is a sequence $\left\langle f_{n}, L f_{n}\right\rangle$ converging to $\langle f, L f\rangle$ in the product space, with each $f_{n}$ in $C_{0}^{\infty}$. It follows from the inequality we just 
proved that $\tau^{i} f_{n}$ is a Cauchy sequence. Since $\left(\tau^{i}\right)_{0}$ is closed, $\tau^{i} f_{n}$ converges to $\tau^{i} f$, and $\tau^{i} f$ is in $L_{2}[0, \infty)$. Passing to the limit, we see that $M(\|f\|+\|L f\|)$ $\geqslant\left\|\tau^{i} f\right\|$. Lemma 4 is proved.

LEMMA 5. Suppose that $\tau$ is a regular formally selfadjoint ordinary differential expression. Let $j$ be a fixed positive integer. Then, for any $N>0$, there is $a \quad \lambda>0$ such that, for any $j$-tuple $\left\langle g_{0}, \cdots, g_{j-1}\right\rangle$ of bounded $C^{\infty}$ functions with $\Sigma_{0}^{j-1}\left\|g_{m}\right\| \leqslant N,\left(\tau^{j}+\Sigma^{j-1} g_{m} \tau^{m}+z \lambda\right)_{0}$ has closed range, where $z= \pm(-1)^{1 / 2}$.

Proof of Lemma 5. If the lemma were not true there would be a sequence $f_{n}$ in $C_{0}^{\infty}$ with $\left\|f_{n}\right\|=1$ and $\left\|\left(L_{n}+n z\right) f_{n}\right\|$ converging to zero, where $L_{n}=\tau^{j}+\Sigma_{m=0}^{j-1} g_{n, m} \tau^{m}$, and $\Sigma_{m=0}^{j-1}\left\|g_{n, m}\right\|_{\infty} \leqslant N$. To see this, note that $L_{0}$ has closed range if and only if $\left(L_{0}\right)^{-1}$ is a bounded linear transformation from range $L_{0}$ into $L_{2}[0, \infty)$, for any $L$, and observe that we may choose the $f_{n}$ in $C_{0}^{\infty}$ since any $L_{0}$ is the closure of its restriction to $C_{0}^{\infty}$.

Let $\left\|\left(\tau^{j}+\Sigma_{0}^{j-1} g_{n, m} \tau^{m}+n z\right) f_{n}\right\|=c_{n}$. We know by hypothesis that $c_{n}$ converges to zero. Since $\left\|n z f_{n}\right\|=n$, it follows that, for some $k \leqslant j$, $\left\|\tau^{k} f_{n_{i}}\right\|$ must converge to infinity for some subsequence $f_{n_{i}}$. Without loss of generality, we may assume that the $f_{n_{i}}$ are the original sequence $f_{n}$, since we will end up by showing that the sequence $f_{n_{i}}$ cannot exist. By Lemma 4, we now see that, for any $m<j,\left(\tau^{2 m} f_{n}, f_{n}\right) /\left(\tau^{2 j} f_{n}, f_{n}\right)$ converges to zero, since $\tau_{0}$ has a selfadjoint extension (perhaps to a larger Hilbert space) and since $\left(\tau^{2 k} f_{n}, f_{n}\right)=\left\|\tau^{k} f_{n}\right\|^{2}$ converges to infinity. Thus $\left\|\tau^{m} f_{n}\right\| /\left\|\tau^{j} f_{n}\right\|$ converges to zero for any $m<j$.

Since

$$
\left\|\tau^{j} f_{n}+\sum_{0}^{j-1} g_{n, m} \tau^{m} f_{n}+n z f_{n}\right\| \geqslant\left\|\tau^{j} f_{n}+n z f_{n}\right\|-N \sum_{0}^{j-1}\left\|\tau^{m} f_{n}\right\|,
$$

we see that $c_{n} /\left\|\tau^{j} f_{n}\right\|$ becomes eventually greater than $\left(\left\|\tau^{j} f_{n}+n z f_{n}\right\| /\left\|\tau^{j} f_{n}\right\|\right)-\epsilon$ for any $\epsilon>0$. Since $c_{n}$ converges to zero, it follows that $\left\|\tau^{j} f_{n}+n z f_{n}\right\| /\left\|\tau^{j} f_{n}\right\|$ converges to zero. But, since

$$
\left\|\tau^{j} f_{n}\right\|-n \leqslant\left\|\tau^{j} f_{n}+n z f_{n}\right\|
$$

it follows from dividing the inequality through by $\left\|\tau^{j} f_{n}\right\|$ that $1-n /\left\|\tau^{j} f_{n}\right\|$ eventually becomes less than any positive $\epsilon$. Also, similarly, $n /\left\|\tau^{j} f_{n}\right\|-1$ becomes less than any positive $\epsilon$, so that we have shown that $n /\left\|\tau^{j} f_{n}\right\|$ converges to 1 . Since 


$$
\left\|\tau^{m} f_{n}\right\| /\left\|\tau^{j} f_{n}\right\|=\left(\left\|\tau^{m} f_{n}\right\| / n\right)\left(n /\left\|\tau^{j} f_{n}\right\|\right)
$$

we see that $\left\|\tau^{m} f_{n}\right\| / n$ converges to zero for every $m<j$.

Since $\left(\tau^{j} f_{n}, f_{n}\right)$ is real, we see by the Schwarz inequality that the imaginary part of $\left(L_{n} f_{n}, f_{n}\right) / n$ converges to zero. Thus the imaginary part of $\left(\left(L_{n}+n z\right) f_{n}, f_{n}\right) / n$ converges to 1 . This contradicts our original assumption that $\left\|\left(L_{n}+n z\right) f_{n}\right\|$ converges to zero. Lemma 5 is proved.

We also need a minor technical lemma.

LEMMA 6. Suppose that $L_{1}$ and $L_{2}$ are regular ordinary differential expressions, and there is an $M>0$ such that, for every $f$ in $C_{0}^{\infty},\left\|L_{2} f\right\| \leqslant$ $M\left(\|f\|+\left\|L_{1} f\right\|\right)$. Then domain $\left(L_{2}\right)_{0} \supseteq \operatorname{domain}\left(L_{1}\right)_{0}$.

Proof of Lemma 6. $\left(L_{1}\right)_{0}$ is the closure of the restriction of $L_{1}$ to $C_{0}^{\infty}$. But, by hypothesis, if $f_{n}$ and $L_{1} f_{n}$ are Cauchy sequences of $C_{0}^{\infty}$ functions, $L_{2} f_{n}$ is a Cauchy sequence. Since $\left(L_{2}\right)_{0}$ is a closed operator, the conclusion follows.

Proof of TheOREM 8. We observe that adding a complex number to a differential expression does not alter the limit point condition, since it does not change the domain of the maximal or minimal operators. We will first show that if $\lambda>0$ is large enough, $\tau^{j}+\Sigma_{0}^{j-1} d_{i} \tau^{i}+\lambda z$ is in the limit point condition, where $z=(-1)^{1 / 2}$. This will show that any polynomial in $\tau$ of degree $j$ is in the limit point condition. From this, the general case will be proved using Lemma 2.

Let $\Sigma_{0}^{j-1}\left|d_{i}\right|=K$. Let $\Sigma_{0}^{j-1}\left|c_{i}\right|=K_{1}$. Set $N=K+K_{1}$, and select, using Lemma $5, \lambda\rangle 0$ such that for all $j$-tuples $\left\langle b_{0}, b_{1}, \cdots, b_{j-1}\right\rangle$ of complex numbers with $\Sigma_{0}^{j-1}\left|b_{i}\right|<N,\left(\tau^{j}+\Sigma_{0}^{j-1} b_{i} \tau^{i}+\lambda z\right)_{0}$ has closed range. By taking adjoints, and using Theorem 1 , it follows that for any such $j$-tuple, $\left(\tau^{j}+\Sigma_{0}^{j-1} b_{i} \tau^{i}-\lambda z\right)_{0}$ also has closed range.

We know by hypothesis that $\tau^{j}+\Sigma_{0}^{j-1} c_{i} \tau^{i}+\lambda z$ is in the limit point condition. Let $L(\theta)$ be the differential expression $\tau^{j}+\Sigma_{0}^{j-1}\left((1-\theta) c_{i}+\theta d_{i}\right) \tau^{i}$ $+\lambda z$, for each $0 \leqslant \theta \leqslant 1$. Let $S$ be the set of all $\theta$ in $[0,1]$ with $L(\theta)$ in the limit point condition. We show that $S$ is both open and closed, and since $L(0)$ is in the limit point condition, it follows that all $L(\theta)$ are in the limit point condition.

Suppose that $\theta \in[0,1]$. We show, using Theorem 6, that nullity $\left((L(\phi))^{+}\right)_{M}$ $=\operatorname{nullity}\left((L(\theta))^{+}\right)_{M}$ for all $\phi$ in a small neighborhood of $\theta$. By Lemma 4 , the restriction of $\tau^{i}$ to domain $(L(\theta))_{0}$ is $(L(\theta))_{0}$ bounded for each $i \leqslant j$. Thus, by Theorem 6, index $(L(\theta))_{0}+\Sigma_{0}^{j-1}\left((\theta-\phi) c_{i}+(\phi-\theta) d_{i}\right) R_{i}$ is constant for $\phi$ close enough to $\theta$, where $R_{i}$ is the restriction of $\tau^{i}$ to domain $(L(\theta))_{0}$. We would like to say that 


$$
(L(\theta))_{0}+\sum_{0}^{j-1}\left((\theta-\phi) c_{i}+(\phi-\theta) d_{i}\right) R_{i}=(L(\phi))_{0}
$$

but this requires a short proof. Set $B=\Sigma_{0}^{j-1}(\theta-\phi)\left(c_{i}-d_{i}\right) R_{i}$. By definition, domain $\left((L(\theta))_{0}+B\right)$ is domain $(L(\theta))_{0}$. By Lemmas 4 and 6 , domain $(L(\theta))_{0}$ is domain $(L(\phi))_{0}$. Thus it follows that $(L(\theta))_{0}+B=(L(\phi))_{0}$, and therefore index $(L(\phi))_{0}=\operatorname{index}\left((L(\theta))_{0}+B\right)=\operatorname{index}(L(\theta))_{0}$. Thus nullity $\left((L(\theta))^{+}\right)_{M}=$ nullity $\left((L(\phi))^{+}\right)_{M}$. By the same argument, interchanging $L^{+}(\theta)$ and $L(\theta)$, it follows that nullity $(L(\theta))_{M}=\operatorname{nullity}(L(\phi))_{M}$ for all $\phi$ in a neighborhood of $\theta$. From Theorem 7, it follows that if either $L(\theta)$ or $L(\phi)$ is in the limit point condition, both $L(\theta)$ and $L(\phi)$ are in the limit point condition. Thus $S$ is open and closed, and so all $L(\theta)$ are in the limit point condition, so, in particular, $\tau^{j}+\Sigma_{0}^{j-1} d_{i} \tau^{i}+\lambda z$ is in the limit point condition. Since addition of the term $\lambda z$ does not alter the limit point condition, we have proved that if one polynomial of degree $j$ in $\tau$ is in the limit point condition, all polynomials of degree $j$ in $\tau$ are in the limit point condition.

We now prove that all polynomials in $\tau$ of degree less than $j$ are also in the limit point condition. By what we just proved $(\tau+z)^{j}$ is in the limit point condition. Since, as has been pointed out in Corollary 1 to Theorem $7,(\tau+z)_{0}$ has closed range, it follows from Lemma 2 that $(\tau+z)^{k}$ is in the limit point condition for any $1 \leqslant k<j$. Therefore any polynomial of degree $k$ in $\tau$ is in the limit point condition. Theorem 8 is proved.

The proof of Theorem 8 actually proves some stronger statements, which we list as corollaries.

Corollary 1. Any two polynomials $L_{1}$ and $L_{2}$ of the same degree in a regular formally selfadjoint ordinary differential expression $\tau$ have the property that $\left(L_{1}\right)_{M}$ and $\left(L_{2}\right)_{M}$ are equal dimensional extensions of their respective minimal operators, whether or not the expressions are in the limit point condition.

COROLlary 2. Any two real polynomials of the same degree in a regular formally selfadjoint ordinary differential expression $\tau$ have the same positive and negative deficiency indices, provided that the leading coefficients of the two polynomials have the same sign. This is true whether or not the positive and negative deficiency indices are equal.

THEOREM 9. Suppose that $\tau$ is a regular formally selfadjoint ordinary differential expression. Suppose that $P(\tau)=\tau^{j}+\Sigma_{0}^{j-1} c_{i} \tau^{i}$ is in the limit point condition, where the $c_{i}$ are complex numbers. Then, if $i \leqslant j, \tau^{i} f$ is in $L_{2}[0, \infty)$ for each $f$ in domain $(P(\tau))_{M}$. 
REMARK. As Lemma 4 shows, this is always true if $(P(\tau))_{M}$ is replaced by $(P(\tau))_{0}$. However, the condition that $\tau^{i} f$ is in $L_{2}[0, \infty)$ for all $f$ in domain $(P(\tau))_{M}$, known as the partial separation condition, occurs if and only if $P(\tau)$ is in the limit point condition, provided that $\tau$ is in the limit point condition. This fact will be established by Theorems 9 and 10 .

Proof of THEOREM 9. It follows from Lemma 4 that, if $f$ is in domain $(P(\tau))_{0}, \tau^{i} f$ is in $L_{2}[0, \infty)$ for all $i \leqslant j$. But if $P(\tau)$ is in the limit point condition, domain $P(\tau)_{M}$ is the vector subspace (which is not closed) generated by domain $(P(\tau))_{0}$ and a set $\left\{f_{m}\right\}_{0}^{j n-1}$ of $j n C^{\infty}$ functions, where $n$ is the order of $\tau$, which vanish outside some interval $[0, N]$ and which have $\left(D^{i} f_{m}\right)(0)=\delta_{i m}$ for all nonnegative integers $i$ and $m$ less than or equal to $\dot{m}-1$. (See the remark following the definition of the limit point condition.) The conclusion follows.

THEOREM 10. Suppose that $\tau$ is a regular formally selfadjoint ordinary differential expression, and that $\tau$ is in the limit point condition. Suppose $P(\tau)$ $=\tau^{j}+\Sigma_{0}^{j-1} c_{i} \tau^{i}$, where all $c_{i}$ are complex numbers. Then if, for every $f$ in domain $(P(\tau))_{M}, \tau^{i} f$ is in $L_{2}[0, \infty)$ for all $i \leqslant j$, it follows that $P(\tau)$ is in the limit point condition.

Proof of Theorem 10. We first show that domain $(P(\tau))_{M}=\operatorname{domain}(Q(\tau))_{M}$ for any other $j$ th degree polynomial $Q(\tau)=\tau^{j}+\Sigma_{0}^{j-1} d_{i} \tau^{i}$. It follows from Lemmas 4 and 6 that domain $(P(\tau))_{0}=\operatorname{domain}(Q(\tau))_{0}$, and by hypothesis domain $(P(\tau))_{M} \subseteq$ domain $(Q(\tau))_{M}$. Thus if $(P(\tau))_{M}$ and $(Q(\tau))_{M}$ are equal dimensional extensions of their associated minimal operators, domain $(P(\tau))_{M}=$ $\operatorname{domain}(Q(\tau))_{M}$.

Using Lemma 5 , we select $\lambda>0$ so large that $((1-\theta) P(\tau)+\theta Q(\tau)+\lambda z)_{0}$ has closed range for all $\theta \in[0,1]$, where $z=(-1)^{1 / 2}$. It then follows from Theorem 1 that $\left((1-\theta)(P(\tau))^{+}+\theta Q(\tau)^{+}-\lambda z\right)_{0}$ also has closed range for all $\theta \in[0,1]$. Since addition of the term $\lambda z$ to a differential expression does not change the domain of either the minimal or maximal operator, we need only show that $(Q(\tau)+\lambda z)_{M}$ and $(P(\tau)+\lambda z)_{M}$ are equal dimensional extensions of their associated minimal operators. It follows from Corollary 1 to Theorem 7 that if nullity $(P(\tau)+\lambda z)_{M}=\operatorname{nullity}(Q(\tau)+\lambda z)_{M}$, and also nullity $\left((P(\tau))^{+}-\lambda z\right)_{M}$ $=\operatorname{nullity}\left((Q(\tau))^{+}-\lambda z\right)_{M}$, then $(Q(\tau)+\lambda z)_{M}$ and $(P(\tau)+\lambda z)_{M}$ are in fact equal dimensional extensions of their associated minimal operators. However, letting $L(\theta)$ denote $\theta Q(\tau)+(1-\theta) P(\tau)+\lambda z$, we see by the method of proof of Theorem 8 that nullity $(L(\theta))_{M}$ is constant for $\theta \in[0,1]$, and also nullity $\left((L(\theta))^{+}\right)_{M}$ is constant for $\theta \in[0,1]$. Thus $\operatorname{domain}(Q(\tau)+\lambda z)_{M}=\operatorname{domain}(P(\tau)+\lambda z)_{M}$.

Now we proceed with the proof of the theorem. By hypothesis, and by what we just proved, it follows that if $f$ is in domain $\left((\tau+z)^{j}\right)_{M}$, then $(\tau+z)^{i} f$ 
is in $L_{2}[0, \infty)$ for any $i \leqslant j$. Therefore, in particular, $(\tau+z) f$ is in $L_{2}[0, \infty)$, so that $f$ is in domain $(\tau+z)_{M}$. Also, however, $(\tau+z)^{2} f$ is in $L_{2}[0, \infty)$, so $(\tau+z) f$ is in domain $(\tau+z)_{M}$. Proceeding in this fashion, we see that $\left((\tau+z)^{j}\right)_{M}$ $=\left((\tau+z)_{M}\right)^{j}$, where, as usual, the product $A B$ of two operators $A$ and $B$ is defined to be $A(B x)$ for each $x$ in domain $A B$, and domain $A B$ is defined to be the set of all $x$ such that $B x$ is in domain $A$.

Since each $(\tau+z)_{0}$ has closed range, it follows that each $(\tau+z)_{M}$ is surjective. Thus nullity $\left((\tau+z)^{j}\right)_{M}=\operatorname{nullity}\left((\tau+z)_{M}\right)^{j}=j \times \operatorname{nullity}(\tau+z)_{M}$. Likewise, we may show that nullity $\left(\left((\tau+z)^{j}\right)^{+}\right)_{M}=\operatorname{nullity}\left((\tau-z)^{j}\right)_{M}=$ $j \times \operatorname{nullity}(\tau-z)_{M}$. Since $\tau$ is in the limit point condition, it follows from Theorem 7 that nullity $(\tau+z)_{M}+\operatorname{nullity}(\tau-z)_{M}=n$, where $n$ is the order of $\tau$. Thus nullity $\left((\tau+z)^{j}\right)_{M}+$ nullity $\left(\left((\tau+z)^{j}\right)^{+}\right)_{M}=j$. But, by Lemma $1,\left((\tau+z)^{j}\right)_{0}$ has closed range. Thus $(\tau+z)^{j}$ is in the limit point condition and therefore, by Theorem $8, P(\tau)$ is in the limit point condition. Theorem 10 is proved.

REMARK. All the theorems of $\S 2$ remain true for vector matrix equations. The extension of the results of $\S 2$ to the vector matrix case is straightforward.

3. Polynomials in first and second order expressions. We now study sufficient conditions for expressions involving powers of first and second order expressions to be in the limit point condition. It is not known to the author whether these theorems generalize to the vector matrix case.

THEOREM 11. Suppose that $V$ is a real valued $C^{\infty}$ function on $[0, \infty)$, which is bounded below. Suppose that, for every nonnegative integer $i \leqslant$ $\max (2 n-4,0)$ there is $a k>0$ such that $e^{-k x} V^{(i)}(x)$ is a bounded function on $[0, \infty)$, where $V^{(i)}$ is the ith derivative of $V$. Let $\tau=-d^{2} / d x^{2}+V$. Then any complex polynomial in $\tau$ of degree $n$ or less is in the limit point condition.

PROof. We first need several lemmas, which will also be useful in the proof of Theorem 12.

LEMma 7. Suppose that $S$ is a dense subspace of a Hilbert space $h$. Suppose that $M$ is a closed subspace with finite deficiency $n$ in $h$. Then $M \cap S$ has deficiency $n$ in $S$. Further, $M \cap S$ is dense in $M$.

Proof of Lemma 7. Clearly, any $n+1$ nonzero vectors in $h$ have a nontrivial linear combination in $M$. If these vectors are all in $S$, then the linear combination is in $M \cap S$. Thus the deficiency of $S \cap M$ in $S$ is at most $n$.

However, suppose that $S_{1}$ is a subspace of $S$ such that $S \cap M+S_{1}=S$, and $S_{1} \cap(S \cap M)$ is the zero vector. Then $M+S_{1} \supseteq S$, so $M+S_{1}$ is 
dense in $h$. Further, since $M$ is a closed subspace with finite deficiency, $M+S_{1}$ is also closed, and therefore $M+S_{1}=h$. Thus the dimension of $S_{1}$ is at least $n$, so the deficiency of $S \cap M$ in $S$ is at least $n$.

If $M \cap S$ were not dense in $M$, there would be a vector $x$ in $M$ which is perpendicular to $M \cap S$. Thus at least $n+1$ linearly independent vectors in $h$ would be perpendicular to $M \cap S$. We have just shown that there is an $n$-dimensional subspace $S_{1}$ of $S$ such that $M \cap S+S_{1}=S$. However, since $(M \cap S)^{\perp}$ is at least $(n+1)$-dimensional, it is easy to see that at least one member of $(M \cap S)^{\perp}$ must be perpendicular to all of $S_{1}$. Thus $M \cap S+S_{1}$ cannot be dense in $h$, a contradiction.

LEMMA 8. Suppose that $\tau_{1}$ and $\tau_{2}$ are regular ordinary differential expressions, and that both $\left(\tau_{1}\right)_{0}$ and $\left(\tau_{2}\right)_{0}$ have closed range. Then the product $\left(\tau_{1}\right)_{0}\left(\tau_{2}\right)_{0}$ has closed range, and its index is index $\left(\tau_{1}\right)_{0}+\operatorname{index}\left(\tau_{2}\right)_{0}$.

Proof. This follows from a deep theorem of Gohberg and Krein, which is discussed on p. 102 of Goldberg [5]. However, since the proof of this special case is quite simple, we include it.

First, $\left(\tau_{1}\right)_{0}\left(\tau_{2}\right)_{0}$ clearly has closed range, because each $\left(\tau_{i}\right)_{0}$ has a bounded inverse from range $\left(\tau_{i}\right)_{0}$ into $L_{2}[0, \infty)$. The deficiency of range $\left(\tau_{1}\right)_{0}\left(\tau_{2}\right)_{0}$ in $L_{2}[0, \infty)$ is the deficiency of range $\left(\tau_{1}\right)_{0}$ in $L_{2}[0, \infty)+$ the deficiency of range $\left(\tau_{2}\right)_{0} \cap$ domain $\left(\tau_{1}\right)_{0}$ in domain $\left(\tau_{1}\right)_{0}$. By Lemma 7 , this last deficiency is the same as the deficiency of range $\left(\tau_{2}\right)_{0}$ in $L_{2}[0, \infty)$. Lemma 8 is proved.

LEMMA 9. Suppose that $\tau_{1}$ and $\tau_{2}$ are regular ordinary differential expressions. Suppose that $\left(\tau_{1}\right)_{0}$ has closed range. Then $\left(\tau_{1} \tau_{2}\right)_{0} \subseteq\left(\tau_{1}\right)_{0}\left(\tau_{2}\right)_{0}$.

Proof. Suppose $f$ is in the domain of $\left(\tau_{1} \tau_{2}\right)_{0}$. Then there is a sequence $\left\{f_{n}\right\}$ of $C_{0}^{\infty}$ functions such that $f_{n}$ converges to $f$, and $\tau_{1} \tau_{2} f_{n}$ converges to $\tau_{1} \tau_{2} f$. Note that while we cannot yet assert that $\tau_{2} f$ is in $L_{2}[0, \infty)$, it is clear that $\tau_{2} f_{n}$ is a Cauchy sequence, since $\tau_{1} \tau_{2} f_{n}$ is Cauchy, $\tau_{2} f_{n}$ is in domain $\left(\tau_{1}\right)_{0}$, and $\left(\tau_{1}\right)_{0}$ has a bounded inverse (by the closed graph theorem) from its range into $L_{2}[0, \infty)$. Thus $\tau_{2} f_{n}$ converges to some $g$ in domain $\left(\tau_{1}\right)_{0}$, and since $\left(\tau_{2}\right)_{0}$ is a closed operator, $g$ must equal $\tau_{2} f$.

Lemma 10. Suppose that $\tau$ is a regular ordinary differential espression, such that $\tau_{0}$ has closed range, and $\tau$ is in the limit point condition. Then $\tau^{j}$ is in the limit point condition if and only if both $\left(\tau^{j}\right)_{0}=\left(\tau_{0}\right)^{j}$, and $\left(\left(\tau^{+}\right)^{j}\right)_{0}=\left(\left(\tau^{+}\right)_{0}\right)^{j}$.

Proof. Index $\left(\tau_{0}\right)^{j}=j \times$ index $\tau_{0}$, by Lemma 8. Since $\tau$ is in the limit point condition, index $\left(\tau_{0}\right)^{j}+$ index $\left(\left(\tau^{+}\right)_{0}\right)^{j}=-j n$, where $n$ is the order of $\tau$, since the index of $\tau_{0}$ is - nullity $\tau_{M}$. Thus, if $\left(\tau^{j}\right)_{0}=\left(\tau_{0}\right)^{j}$ and $\left(\left(\tau^{+}\right)^{j}\right)_{0}=\left(\left(\tau^{+}\right)_{0}\right)^{j}, \tau^{j}$ is in the limit point condition. 
If, however, $\tau^{j}$ is in the limit point condition, then index $\left(\tau^{j}\right)_{0}+$ index $\left(\left(\tau^{+}\right)^{j}\right)_{0}=-j n$. Since $\left(\tau^{j}\right)_{0} \subseteq\left(\tau_{0}\right)^{j}$, and $\left(\left(\tau^{+}\right)^{j}\right)_{0} \subseteq\left(\left(\tau^{+}\right)_{0}\right)^{j}$, it follows that index $\left(\tau^{j}\right)_{0} \leqslant$ index $\left(\tau_{0}\right)^{j}$, with equality holding only if the two operators are equal, and the same relationship holds for $\tau^{+}$. Therefore if index $\left(\tau^{j}\right)_{0}$ + index $\left(\left(\tau^{+}\right)^{j}\right)_{0}$ is as large as $-j n$, it follows that $\left(\tau^{j}\right)_{0}=\left(\tau_{0}\right)^{j}$, and $\left(\left(\tau^{+}\right)^{j}\right)_{0}=\left(\left(\tau^{+}\right)_{0}\right)^{j}$, since index $\left(\left(\tau^{+}\right)_{0}\right)^{j}+$ index $\left(\tau_{0}\right)^{j}=-j n$. Lemma 10 is proved.

Proof OF THEOREM 11. We prove Theorem 11 first for a special case. Suppose that $\tau=-d^{2} / d x^{2}+V$, where $V(x) \geqslant c>0$ for some positive real number $c$, and where $V \in C^{\infty}$. Suppose further that there is a monotonically increasing positive solution $f$ to the equation $\tau f=0$ with the property that $(1 / f(x-1)) R(x)$ approaches zero as $x$ approaches infinity, where $R(x)=$ $4^{n-1}\left(1+\Sigma_{0}^{2 n-4}\left|V^{(i)}(x)\right|\right)^{n-1}$, and $V^{(i)}$ denotes the $i$ th derivative of $V$. Note that, for any $j \leqslant n-1, R(x) \geqslant g_{i}(x)$ for every $g_{i}$ and every $x$, where $\tau^{j}=$ $\sum_{i=0}^{2 j} g_{i} D^{i}$. To see this, note that each $g_{i}$ is a finite sum of terms of type

$$
V^{(\alpha(1))} \times V^{(\alpha(2))} \times \cdots \times V^{(\alpha(j))} \text {, where } \sum_{k=1}^{j} \alpha(k) \leqslant 2 j-2 .
$$

So each $g_{i}$ is a finite sum of terms which occur in $\left(1+\Sigma_{0}^{2 n-4} V^{(i)}\right)^{n-1}$. Also, it is easily seen that, even before any terms are collected, there are less than $4^{j}$ terms which arise when $\left(D^{2}+V\right)^{j}$ is multiplied out. The estimate follows.

We wish to show that $\tau^{n}$ is in the limit point condition, for then, using Theorem 8, we can assert that any polynomial of degree $n$ or less in $\tau$ is in the limit point condition. Note that, if $g \in C_{0}^{\infty},(\tau g, g) \geqslant c(g, g)$, so, by passing to the closure, we see that $\left(\tau_{0}\right)^{-1}$ is a bounded transformation from range $\tau_{0}$ into $L_{2}[0, \infty)$. Since $\tau_{0}$ is a closed operator, it follows that range $\tau_{0}$ is closed. It follows from Corollary 3 to Theorem 7 that there is at least a one-dimensional space $K$ of solutions to the equation $\tau g=0$ which lie in $L_{2}[0, \infty)$. Since the monotonically increasing solution $f$ hypothesized above is clearly not in $L_{2}[0, \infty), K$ is exactly one-dimensional. Therefore $\tau$ is in the limit point condition (as is well known from the classical theory).

By Lemma 10, we need only to show that $\left(\tau^{n}\right)_{0}=\left(\tau_{0}\right)^{n}$. Let us suppose that $\left(\tau^{j}\right)_{0}=\left(\tau_{0}\right)^{j}$ for all $j \leqslant N \leqslant n-1$. We attempt to show that $\left(\tau^{N+1}\right)_{0}$ $=\left(\tau_{0}\right)^{N+1}$.

By Lemma $8,\left(\tau^{N+1}\right)_{0} \subseteq\left(\tau_{0}\right)^{N+1}$. Suppose that $\left\langle\phi, \tau^{N+1} \phi\right\rangle$ is in $\operatorname{graph}\left(\tau_{0}\right)^{N+1}$. Given $\epsilon>0$, we need a $C_{0}^{\infty}$ function $g$ such that $\left\langle g, \tau^{N+1} g\right\rangle$ is within $\epsilon$ of $\left\langle\phi, \tau^{N+1} \phi\right\rangle$ in the product space $L_{2}[0, \infty) \times L_{2}[0, \infty)$. Given $\epsilon_{1}>0$, there is a $C_{0}^{\infty}$ function $h$ such that $\left\langle h, \tau^{N} h\right\rangle$ is within $\epsilon_{1}$ of $\left\langle\tau \phi, \tau^{N+1} \phi\right\rangle$ in the product space, by the induction hypothesis. The difficulty is that $h$ may not be the image under $\tau$ of a $C_{0}^{\infty}$ function. However, if for each $\epsilon_{1}>0$ we could choose an $h$ in $C_{0}^{\infty}$ with $\left\langle h, \tau^{N} h\right\rangle$ within $\epsilon_{1}$ of $\left\langle\tau \phi, \tau^{N+1} \phi\right\rangle$, 
and in addition $\int_{0}^{\infty} h(x) \bar{f}_{i}(x) d x=0$ for each solution $f_{i}$ of $\tau f_{i}=0$, it would follow that $h=\tau g$ for some $g$ in $C_{0}^{\infty}$, as will be shown below. Thus, using the boundedness of $\tau_{0}^{-1}$ as a linear transformation from range $\tau_{0}$ into $L_{2}[0, \infty)$, we would be able to get $\left\langle g, \tau^{N+1} g\right\rangle$ as close as desired to $\left\langle\phi, \tau^{N+1} \phi\right\rangle$, with $g \in C_{0}^{\infty}$. Thus graph $\left(\tau^{j}\right)_{0}$ would be dense in $\operatorname{graph}\left(\tau_{0}\right)^{j}$, so the two operators would be equal, and the special case would be proved.

Therefore we first need to show that if $\int_{0}^{\infty} h(x) \bar{f}_{i}(x) d x=0$ for all solutions $f_{i}$ to the equation $\tau f_{i}=0$, then $h=\tau g$ for some $g \in C_{0}^{\infty}$. If $h$ is supported in $[a, b]$ with $\int_{0}^{\infty} h(x) \bar{f}_{i}(x) d x=0$ for every solution $f_{i}$ to the equation $\tau f_{i}=0$, then $h$ is in the range of the minimal operator corresponding to $\tau$ on $[a, b]$. Therefore $h=\tau g$ on $[a, b]$, and both $g$ and its derivative vanish at $a$ and $b$. Setting $g=0$ outside $[a, b]$, we have $h=\tau g$ on $[0, \infty)$, and $g$ is in $C^{\infty}$ since $h$ is. Thus $h=\tau g$ for $g \in C_{0}^{\infty}$, as we wished to show.

To complete the proof of the special case of Theorem 11, we need only to approximate $\left\langle\tau \phi, \tau^{N+1} \phi\right\rangle$ arbitrarily closely in the product space $L_{2}[0, \infty) \times$ $L_{2}[0, \infty)$ by $\left\langle h, \tau^{N} h\right\rangle$, where $h$ is in $C_{0}^{\infty}$, and $\int_{0}^{\infty} h(x) \bar{f}_{i}(x) d x=0$ for every solution $f_{i}$ to the equation $\tau f_{i}=0$.

Let $K$, as above, be the one-dimensional space of solutions to the equation $\tau g=0$ which lie in $L_{2}[0, \infty)$. We may without difficulty select $\left\langle h, \tau^{N} h\right\rangle$ as close as desired to $\left\langle\tau \phi, \tau^{N+1} \phi\right\rangle$ such that $h$ is in $C_{0}^{\infty}$ and $h \in K^{\perp}$. In fact, the set $S$ of all $\left\langle h, \tau^{N} h\right\rangle$ with $h$ in $C_{0}^{\infty}$ is dense in the Hilbert space formed by considering graph $\left(\tau_{0}\right)^{N}$ as a subspace of the product space $L_{2}[0, \infty) \times$ $L_{2}[0, \infty)$. Also, the set $M$ of all $\left\langle\theta, \tau^{N} \theta\right\rangle$ in $\operatorname{graph}\left(\tau_{0}\right)^{N}$ with $\theta \in K^{\perp}$ is a closed subspace of graph $\left(\tau_{0}\right)^{N}$, since $\left(\left(\tau_{0}\right)^{N}\right)^{-1}$ is a continuous linear transformation from range $\left(\tau_{0}\right)^{N}$ into $L_{2}[0, \infty)$. Since $K^{\perp}$ has deficiency 1 in $L_{2}[0, \infty)$, it follows that $K^{\perp} \cap$ domain $\left(\tau_{0}\right)^{N}$ has deficiency 1 in domain $\left(\tau_{0}\right)^{N}$, by Lemma 7. Thus $M$ has deficiency 1 in $\operatorname{graph}\left(\tau_{0}\right)^{N}$, so that, using Lemma 7 again, $M \cap S$ is dense in $M$. But, since $\phi$ was originally assumed in domain $\left(\tau_{0}\right)^{N+1}, \tau \phi$ is in range $\tau_{0}$, and thus $\tau \phi \in K^{\perp}$.

Thus we can approximate $\left\langle\tau \phi, \tau^{N+1} \phi\right\rangle$, as closely as desired in the product space, by $\left\langle h, \tau^{N} h\right\rangle$, with $h \in C_{0}^{\infty} \cap K^{\perp}$.

We would like to make the same sort of argument for the unbounded solution $f$ such that $f$ is monotonically increasing and positive, and $(1 / f(x-1)) R(x)$ approaches zero as $x$ approaches infinity. Let $S_{1}$ be the set of all $\left\langle h, \tau^{N} h\right\rangle$ with $h$ in $C_{0}^{\infty}$ and $\int_{0}^{\infty} h(x) f(x) d x=0$. We show directly that $S_{1}$ is dense in $\operatorname{graph}\left(\tau_{0}\right)^{N}$ $=\operatorname{graph}\left(\tau^{N}\right)_{0}$.

If $\left\langle h_{1}, \tau^{N} h_{1}\right\rangle$ could be approximated arbitrarily closely in the product space by elements of $S_{1}$, for any $h_{1}$ in $C_{0}^{\infty}$, then $S_{1}$ would be dense in graph $\left(\tau^{N}\right)_{0}$. Let $\gamma(x)$ be a nonnegative, real valued, $C_{0}^{\infty}$ function, defined on 
the whole real line and vanishing outside $[0,1]$, such that $\int_{0}^{1} \gamma(x) d x=1$. Let $\gamma_{t}(x)$ denote $\gamma(x-t)$. Then $\int_{0}^{\infty} f(x) \gamma_{t}(x) d x \geqslant f(t)$. Let $c=\int_{0}^{\infty} h_{1}(x) f(x) d x$. Let $c_{t}=\int_{0}^{\infty} f(x) \gamma_{t}(x) d x$. Then

$$
\int_{0}^{\infty}\left(h_{1}(x)-\left(c / c_{t}\right) \gamma_{t}(x)\right) f(x) d x=0 .
$$

Thus $\left\langle h_{1}-\left(c / c_{t}\right) \gamma_{t}, \tau^{N}\left(h_{1}-\left(c / c_{t}\right) \gamma_{t}\right)\right\rangle$ is in $S_{1}$. If $t$ is taken large enough $\left(c / c_{t}\right) \gamma_{t}$ can be made as close to zero as desired in $L_{2}$ norm. Also

$$
\tau^{N}\left(\left(c / c_{t}\right) \gamma_{t}\right)=\left(c / c_{t}\right)\left((-1)^{N} D^{2 N} \gamma_{t}+\sum_{0}^{2 N-1} g_{i} D^{i} \gamma_{t}\right)
$$

But

$$
\left\|\tau^{N}\left(\left(c / c_{t}\right) \gamma_{t}\right)\right\| \leqslant\left(c / c_{t}\right)\left(\left\|D^{2 N} \gamma\right\|+\sum_{0}^{2 N-1} M_{i}(t)\left\|D^{i} \gamma\right\|\right),
$$

where $M_{i}(t)=\sup \left|g_{i}(x)\right|$, and the supremum is taken over all $x$ in $[t, t+1]$. Also,

$$
M_{i}(t) / c_{t} \leqslant M_{i}(t) / f(t)=\left|g_{i}\left(x_{0}\right)\right| / f(t) \leqslant\left|g_{i}\left(x_{0}\right)\right| / f\left(x_{0}-1\right)
$$

for some $x_{0} \in[t, t+1]$. Therefore, since $\left|g_{i}\left(x_{0}\right)\right| / f\left(x_{0}-1\right)$ converges to zero as $x_{0}$ approaches infinity, it follows that if $t$ is made large enough, $\tau^{N}\left(\left(c / c_{t}\right) \gamma_{t}\right)$ can be made arbitrarily small. Thus $\left\langle\left(c / c_{t}\right) \gamma_{t}, \tau^{N}\left(\left(c / c_{t}\right) \gamma_{t}\right)\right\rangle$ can be made as small as desired in the product space. Since

$$
\int_{0}^{\infty}\left(h_{1}(x)-\left(c / c_{t}\right) \gamma_{t}(x)\right) f(x) d x=0,
$$

this shows that $S_{1}$ is dense in the graph of $\left(\tau^{N}\right)_{0}$.

Now let $M$ be as above. Since $S_{1}$ is dense in the graph of $\left(\tau_{0}\right)^{N}$, and since $M$ is a closed subspace with deficiency 1 in $\operatorname{graph}\left(\tau_{0}\right)^{N}$, it follows that $S_{1} \cap M$ is dense in $M$. This is what we wished to show. The special case of Theorem 11 is proved.

We now prove the general case. Suppose that $V_{1}=V+\lambda$. We will choose $\lambda$ so that $-D^{2}+V_{1}$ satisfies the hypotheses of the special case. This will show that $(\tau+\lambda)^{n}$ is in the limit point condition, and using Theorem 8 we can then assert that $\tau^{n}$ is in the limit point condition.

Note that, if $R_{1}(x)=4^{n-1}\left(1+\Sigma_{0}^{2 n-4}\left|V_{1}^{(i)}(x)\right|\right)^{n-1}$, we can see that $R_{1}(x) \leqslant$ $(1+\lambda)^{n-1} R(x)$ for any $\lambda>0$. Furthermore, $R(x) \leqslant c e^{k x}$ for some $k>0$ and some positive constant $C$. Select $\lambda$ so large that $V(x)+\lambda \geqslant(k+1)^{2}$ for all $\lambda \in[0, \infty)$. Let $f$ be the solution to $(\tau+\lambda) f=0$ with $f(0)=1$ and $f^{\prime}(0)=k+1$. A simple proof is given in Read [9] that $f(x) \geqslant e^{(k+1) x}$ for all $x \in[0, \infty)$. To see that $f$ is monotone increasing, observe that $D^{2} f=$ 
$(V+\lambda) f$, with $V+\lambda \geqslant(k+1)^{2}>0$. Since $f^{\prime}(0)>0$ and $f(0)=1$, we see that if $f^{\prime}\left(x_{0}\right)=0$, there must be a point $x_{1}<x_{0}$ with $D^{2} f\left(x_{1}\right)<0$, so that $f\left(x_{1}\right)<0$. However, $f\left(x_{1}\right)$ cannot be less than zero unless there is an $x_{2}<x_{1}$ with $f^{\prime}\left(x_{2}\right)<0$. Thus there can be no least point $x_{0}$ such that $f^{\prime}\left(x_{0}\right)=0$, and therefore $f^{\prime}$ never takes on the value zero at all. It follows that $f^{\prime}$ is always positive and $f$ is monotone increasing. Thus $f$ satisfies the hypotheses necessary for the special case, and Theorem 11 is proved.

REMARK. The proof of Theorem 11 was really made for any $V$ such that $V+\lambda$ satisfies the hypotheses of the special case for some $\lambda$. Thus a somewhat larger class of functions $V$ than those given by Theorem 11 can be shown, by the proof of Theorem 11, to have the property that $\left(-D^{2}+V\right)^{n}$ is in the limit point condition.

REMARK. It is interesting to compare the hypothesis of Theorem 11 with the condition that $g g^{\prime \prime}$ be in $L_{1}\left[x_{0}, \infty\right)$ for some $x_{0}$, where $g=V^{-1 / 4}$. This is the essential condition of Everitt and Giertz to guarantee that $\left(-D^{2}+V\right)^{n}$ be in the limit point condition. (They also assume that $V(x) \geqslant k>0$.) Their condition only involves $V$ and its second derivative, whereas ours involve $2 n-4$ derivatives. However, as is mentioned on p. 159 of [3], their condition implies that $g(x) g^{\prime}(x)$ approaches zero as $x$ approaches infinity, so that a function such as $V(x)=\left(2+\sin \left(e^{x}\right)\right)^{4}$ satisfies our condition but not theirs. This sort of $V$ is interesting, because the coefficients of powers of $D$ in polynomials in $-D^{2}+V$ become highly unbounded, and it seems to the author that it would be quite difficult to use existing methods other than Theorem 11 to show that all polynonials in $-D^{2}+V$ are in the limit point condition, even if only real polynomials are considered.

Thus it seems that the conditions of Everitt and Giertz and the conditions of the author each apply to functions $V$ which are not covered by the other conditions.

THEOREM 12. Let $z=(-1)^{1 / 2}$. Let $\tau=z D+V$, where $V$ is real valued, $C^{\infty}$, and where there is a $k>0$ such that $e^{-k x} V^{(i)}(x)$ is a bounded function on $[0, \infty)$, for all nonnegative integers $i \leqslant \max (j-2,0)$ where $V^{(i)}$ denotes the ith derivative of $V$. Let $\left\{f_{m}\right\}_{0}^{j-1}$ be a set of $C^{\infty}$ complex valued functions with $f_{m}^{(i)}$ bounded for $0 \leqslant i \leqslant m$. Then $\tau^{j}+\Sigma_{0}^{j-1} f_{m} \tau^{m}$ is in the limit point condition.

Proof. We first must show that $\tau^{j}$ is in the limit point condition. The proof of this fact is similar to that of the preceding theorem, but there are certain differences. We will discuss the differences and merely outline the similar parts.

A solution of $(\tau+z \lambda) f=0$ is $f(x)=\exp \left(-\int_{0}^{x}(z V(t)+\lambda) d t\right)$. Since $|f(x)|=e^{-\lambda x}, f$ is in $L_{2}[0, \infty)$ if and only if $\lambda>0$. Since $(\tau+z \lambda)^{+}=$ 
$\tau-z \lambda$, and since $(\tau+z \lambda)_{0}$ has closed range (because $\tau_{0}$ is symmetric), it follows from Theorem 7 that $\tau+z \lambda$ is in the limit point condition. Let $\lambda=$ $k+1$, where $k$ is given by the hypotheses of the theorem. We first sketch a proof that $\left((\tau+z \lambda)_{0}\right)^{j}=\left((\tau+z \lambda)^{j}\right)_{0}$. Since $\tau+z \lambda$ is not formally selfadjoint, we must then prove that $\left((\tau-z \lambda)_{0}\right)^{j}=\left((\tau-z \lambda)^{j}\right)_{0}$ before we can use Lemma 10 to prove that $(\tau+z \lambda)^{j}$ is in the limit point condition.

Suppose that $\left((\tau+z \lambda)^{N}\right)_{0}=\left((\tau+z \lambda)_{0}\right)^{N}$ for some $N \leqslant j-1$. We prove that $\left((\tau+z \lambda)^{N+1}\right)_{0}=\left((\tau+z \lambda)_{0}\right)^{N+1}$. Let $\left\langle g,(\tau+z \lambda)^{N+1} g\right\rangle$ be in $\operatorname{graph}\left((\tau+z \lambda)_{0}\right)^{N+1}$. Then $\left\langle(\tau+z \lambda) g,(\tau+z \lambda)^{N}(\tau+z \lambda) g\right\rangle$ is in $\operatorname{graph}\left((\tau+z \lambda)_{0}\right)^{N}=\left((\tau+z \lambda)^{N}\right)_{0}$. Let $h_{1}=(\tau+z \lambda) g$. If we can approximate $\left\langle h_{1},(\tau+z \lambda)^{N} h_{1}\right\rangle$ arbitrarily closely by $\langle h,(\tau+z \lambda) h\rangle$, with $h$ in $C_{0}^{\infty}$ and $\int_{0}^{\infty} f(x) \bar{h}(x) d x=0$ for the solution $f(x)$ to the equation $(\tau-z \lambda) f=0$, then it follows as in Theorem 11 that $\left((\tau+z \lambda)^{N+1}\right)_{0}=\left((\tau+z \lambda)_{0}\right)^{N+1}$. As we have seen, all such $f$ have $|f(x)|=c e^{\lambda x}$ for some $c>0$. Exactly the same method as in Theorem 11 allows us to find $h$, so we have proven that $\left((\tau+z \lambda)_{0}\right)^{N+1}=\left((\tau+z \lambda)^{N+1}\right)_{0}$. By induction, we see that $\left((\tau+z \lambda)_{0}\right)^{j}=$ $\left((\tau+z \lambda)^{j}\right)_{0}$.

A different method must be used to prove that $\left((\tau-z \lambda)^{j}\right)_{0}=\left((\tau-z \lambda)_{0}\right)^{j}$. $\left.\left((\tau-z \lambda)_{0}\right)^{j}\right)^{+}=(\tau+z \lambda)^{j}$, and since $(\tau+z \lambda)_{M}$ is surjective (because the minimal operator has closed range), and also the equation $(\tau+z \lambda) f=0$ has all its solutions in $L_{2}[0, \infty)$, it follows that all $j$ linearly independent solutions to the equation $(\tau+z \lambda)^{j} f=0$ are in $L_{2}[0, \infty)$. Thus index $\left((\tau+z \lambda)^{j}\right)_{M}=j$. Therefore index $\left((\tau-z \lambda)^{j}\right)_{0}=-j$. But, by Lemma 8, index $\left((\tau-z \lambda)_{0}\right)^{j}=-j$. Thus, since, by Lemma $9,\left((\tau-z \lambda)_{0}\right)^{j} \supseteq\left((\tau-z \lambda)^{j}\right)_{0}$, it follows that the two operators are equal. We can now use Lemma 10 to say that $(\tau+z \lambda)^{j}$ is in the limit point condition. Then, by Theorem 8 , we know that $\tau^{j}$ is in the limit point condition.

Let $L=\tau^{j}+\Sigma_{0}^{j-1} f_{m} \tau^{m}$. Then

$$
L^{+}=\tau^{j}+\sum_{m=0}^{j-1}\left(\sum_{i=0}^{m} a_{i} f_{m}^{(i)} \tau^{m-i}\right),
$$

where the $a_{i}$ are constant. Thus $L^{+}$is of the form $\tau^{j}+\Sigma_{0}^{j-1} h_{m} \tau^{m}$, where the $h_{m}$ are bounded $C^{\infty}$ functions. Let

$$
N=\sum_{0}^{j-1}\left(\left\|f_{m}\right\|_{\infty}+\left\|h_{m}\right\|_{\infty}\right)
$$

Using Lemma 5 , select $\lambda>0$ so large that both $\left(\tau^{j}+\Sigma_{0}^{j-1} g_{m} \tau^{m}+z \lambda\right)_{0}$ and $\left(\tau^{j}+\Sigma_{0}^{j-1} g_{m} \tau^{m}-z \lambda\right)_{0}$ have closed range for all bounded $C^{\infty}$ functions $g_{m}$ such that $\Sigma_{0}^{j-1}\left\|g_{m}\right\|_{\infty} \leqslant N$. Using Theorem 6 exactly as in the proof of 
Theorem 8 , we see that all minimal operators $\left(\tau^{j}+\Sigma_{0}^{j-1} g_{m} \tau^{m}+z \lambda\right)_{0}$, with $\Sigma_{0}^{j-1}\left\|g_{m}\right\|_{\infty} \leqslant N$, have the same index. Also, all minimal operators of type $\left(\tau^{j}+\Sigma_{0}^{j-1} g_{m} \tau^{m}-z \lambda\right)_{0}$, with $\Sigma_{0}^{j-1}\left\|g_{m}\right\|_{\infty} \leqslant N$, have the same index. Thus index $(L+z \lambda)_{0}=$ index $\left(\tau^{j}+z \lambda\right)_{0}$, and index $\left(L^{+}-z \lambda\right)_{0}=\operatorname{index}\left(\tau^{j}-z \lambda\right)_{0}$. Therefore $L$ is in the limit point condition, since the index of a minimal operator with closed range is minus the nullity of the maximal operator associated with the adjoint expression. Theorem 12 is proved.

Note added in proof. T. T. Read (Proc. London Math. Soc. (to appear)) has recently generalized Theorem 11 by weakening the hypotheses on the coefficients.

\section{REFERENCES}

1. J. Chaudhuri and W. N. Everitt, On the square of a formally self-adjoint differential expression, J. London Math. Soc. (2) 1 (1969), 661-673. MR 40 \#1814.

2. N. Dunford and J. T. Schwartz, Linear operators. II: Spectral theory. Selfadjoint operators in Hilbert space, Interscience, New York, 1963. MR 32 \#6181.

3. W. N. Everitt and M. Giertz, On some properties of the powers of a formally selfadjoint differential expression, Proc. London Math. Soc. (3) 24 (1972), 149-170.

4. I. C. Gohberg and M. G. Krein, The basic propositions on defect numbers, root numbers, and indices of linear operators, Uspehi Mat. Nauk 12 (1957), no. 2 (74), 43-11 8; English transl., Amer. Math. Soc. Transl. (2) 13 (1960), 185-264. MR 20 \#3459; 22 \#3984.

5. S. Goldberg, Unbounded linear operators: Theory and applications, McGraw-Hill, New York, 1966. MR 34 \#580.

6. T. Kato, Perturbation theory for nullity, deficiency, and other quantities of linear operators, J. Analyse Math. 6 (1958), 261-322. MR 21 \#6541.

7. R. M. Kauffman, Compactness of the inverse of the minimal operator for a class of ordinary differential expressions, J. Reine Angew. Math. 257 (1972), 91-99. MR 46 \#9802.

8. M. A. NaImark, Linear differential operators, GITTL, Moscow, 1954; English transl., Part II, Ungar, New York, 1968. MR 16, 702; 41 \#7485.

9. T. T. Read, Growth and decay of solutions of $y^{(2 n)}-p y=0$, Proc. Amer. Math. Soc. 43 (1974), 127-132.

10. A. Zettl, The limit point and limit circle cases for polynomials in a differential operator, Proc. Roy. Soc. Edinburgh (to appear).

DEPARTMENT OF MATHEMATICS AND COMPUTER SCIENCE, WESTERN WASHINGTON STATE COLLEGE, BELLINGHAM, WASHINGTON 98225

Current address: Department of Mathematics, University of Dundee, Dundee, Scotland 